\title{
Laparoscopic TVT Sling Removal Following Prior Transvaginal Sling Excision
}

\author{
Sagarika Sinha, DO, John R. Miklos, MD, Robert D. Moore, DO \\ Atlanta Urogynecology Associates, Alpharetta, GA, USA (all authors).
}

\begin{abstract}
Several studies have reported the incidence and prevalence of retropubic tension-free vaginal mesh tape (TVT) slingrelated complications, but there are no specific current guidelines regarding the surgical management of such complications. Complications requiring sling removal, such as pain, can be surgically managed by using either a vaginal approach, removing the vaginal portion of the sling up to the pubocervical fascia, and/or a laparoscopic/abdominal approach to the space of Retzius to remove the retropubic portion of the sling. We report a case of a patient with a retropubic TVT sling in whom the removal of the vaginal portion of the sling failed to resolve the patient's symptoms of pain; therefore, a laparoscopic approach to the retropubic space was performed to extract the remaining limbs of the sling.
\end{abstract}

Key Words: TVT sling, Mesh complication, vaginal mesh, Laparoscopic, Stress Urinary Incontinence.

Citation Sinha S, Miklos JR, Moore RD. Laparoscopic TVT sling removal following prior transvaginal sling excision. CRSLS e2014.00095. DOI: 10.4293/CRSLS.2014.00095.

Copyright $\odot 2014$ SLS This is an open-access article distributed under the terms of the Creative Commons Attribution-Noncommercial-ShareAlike 3.0 Unported license which permits unrestricted noncommercial use, distribution, and reproduction in any medium, provided the original author and source are credited.

Address correspondence to: Robert D. Moore, DO, Atlanta Urogynecology Associates, 3400 Old Milton Pkwy, Building C, Suite 330, Alpharetta, GA 30005, USA Telephone: (770) 475-4499, Fax: (678) 262-3671, E-mail: moorer33@hotmail.com

\section{INTRODUCTION}

Retropubic mesh tape slings such as the tension-free vaginal tape (TVT) sling (Gynecare, Somerville, New Jersey, USA) and its analogues have become one of the first-line surgical therapies for stress urinary incontinence secondary to its successful outcomes and low complication rates. ${ }^{1}$ More than 1 million TVT slings have been placed worldwide since their first inception in 1995 by Ulmsten and Petros. ${ }^{2}$ Overall complication rates are very low; however, potential complications that have been reported are chronic pelvic pain, dyspareunia, mesh erosions into the bladder, extrusion into the vagina, recurrent urinary tract infections, urinary obstructive symptoms, and de novo detrusor instability. ${ }^{1-3}$ Some of these complications are refractory to conservative treatment and may require surgical management. There are limited reports on surgical management of these complications in the current literature. Transvaginal sling excision/removal and complete laparoscopic TVT resections/removal have been reported separately as management options for some of the complications. ${ }^{3-6}$ In this article, we report one of the first cases of a laparoscopic approach for subsequent removal of the retropubic portion of the TVT sling in a patient with persistent pain after removal of the vaginal portion failed to resolve her symptoms.

\section{CASE REPORT}

A 35year-old woman diagnosed with stress urinary incontinence was treated at another facility with a retropubic TVT sling after which she suffered numerous bladder and vaginal infections, suprapubic pain, lower abdominal pain, vaginal pain, and dyspareunia. Eighteen months postoperatively, the patient was found to have a 3-mm vaginal mesh exposure to the right of the midurethra. Secondary to these symptoms, she underwent a partial TVT sling excision, where the vaginal portion of the sling was removed to the level of the pubocervical fascia bilaterally. Her symptoms remained unresolved after sling excision. Additionally, she then complained of lower abdominal pain with activity, especially with lifting heavy objects. A year later, she was evaluated at our specialty center. On examination, vaginal palpation of the sling retropubically reproduced her pain, and after informed consent, she opted to remove the remaining retropubic portion of the TVT sling via a laparoscopic approach.

The patient was taken to the operating room, where a vertical midline skin incision was made suburetherally in the anterior vaginal wall in attempt to locate any vaginal 


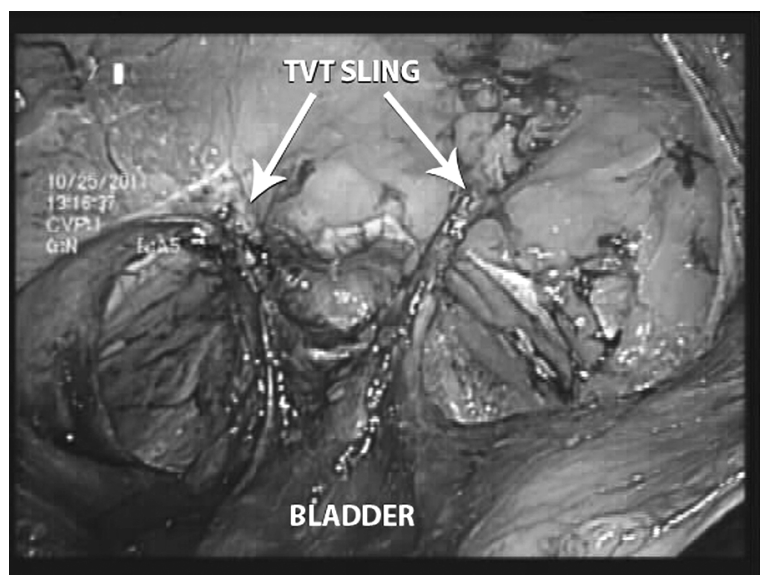

Figure 1. Laparoscopic retropubic view of TVT sling following initial dissection.

portion of the sling left behind. Vaginal epithelium was mobilized bilaterally until the edges of the sling were visualized/palpated under the right and left ischiopubic rami. The mesh was freed in this area and further dissected to the level of the pubocervical fascia. Edges of the sling were clamped for delivery into the space of Retzius for a later point of the procedure.

The bladder was filled to $250 \mathrm{~mL}$; the vesicoperitoneal reflection was identified and incised between the obliterated umbilical ligaments; and the retropubic space was opened up. The sling was identified and visualized running from the pubocervical fascia up to the abdominal wall along the posterior aspect of the pubic symphysis (Figures 1 and 2). The mesh that entered the anterior abdominal wall on the patient's right side was then excised and mobilized all the way down from the iliopectineal line,

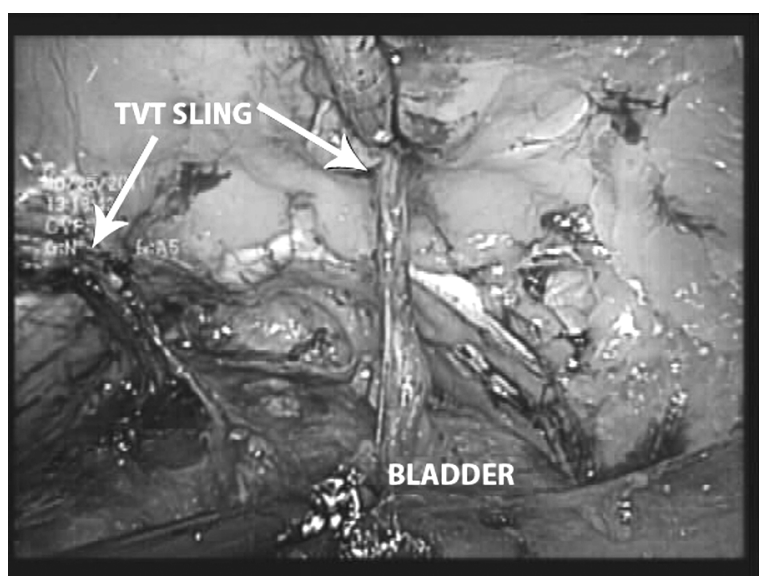

Figure 2. Retropubic view showing TVT mesh tape sling from the abdominal wall down to the pubocervical fascia. off the posterior aspect of the pubic bone and into the pubocervical fascia. A defect was created in the pubocervical fascia around the mesh so that it could be freed up, delivered from below, and removed by pulling it up through the space of Retzius (Figure 3). The same surgical procedure was performed on the patient's left side to remove the mesh in its entirety. The paravaginal defects that were created were then repaired laparoscopically using interrupted sutures, and the vaginal incision was closed from below. Cystoscopy was completed at the end of the case to ensure ureteral patency and no injury to the bladder or urethra.

The patient was discharged home on postoperative day 1 , and her recovery was unremarkable and without complications. On postoperative day 20, the patient noted that her urinary symptoms had improved; she was no longer experiencing urinary incontinence. She was also undergoing pelvic floor physical therapy as part of her recovery. At 5 months following surgery, the patient reported her pain was significantly improved, and her urinary symptoms were 90\% improved in comparison with her symptoms before surgery. She denied any recurrent Stress Urinary Incontinence symptoms and reported improvement in her urge leakage. She was very satisfied with the outcome of the procedure.

\section{DISCUSSION}

The incidence of chronic pelvic pain after placement of a suburetheral sling has been reported to be from $0 \%$ to $30 \%{ }^{4}$ Chronic pain lasting for $>6$ months is found in $1 \%$ of patients who have had a retropubic suburethral sling 7,8 . Although the pathogenesis of pain after TVT placement remains poorly elucidated, removal of the vaginal portion of the sling may resolve

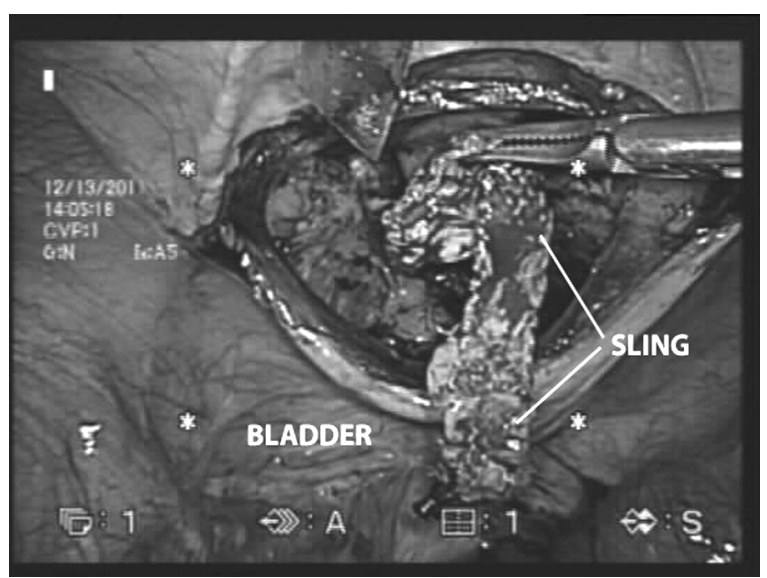

Figure 3. One side of the retropubic portion of the TVT mesh tape sling being removed. 
issues such as vaginal pain alone, dyspareunia, and urinary obstructive symptoms, and in many cases the patient will stay continent even after vaginal resection/removal of the mesh. ${ }^{9}$

Prosthetic material has been associated with the risk of periprosthetic traction phenomenon and inflammatory reactions that may be responsible for the pain secondary to contraction and pulling on the abdominal wall or vaginal tissues, ${ }^{4,7}$ In a patient who presents with lower abdominal pain with activity and/or intercourse that is reproducible on examination with palpation of the sling, consideration needs to be given for removal of both the vaginal and retropubic portions of the mesh. Many clinicians may attempt vaginal removal alone for first-line therapy secondary to the minimally invasive nature of the procedure; however, if the pain and symptoms are not resolved, then consideration should be given to removal of the retropubic portion of the sling.

Abdominal/retropubic removal of a TVT sling is a complex procedure; however, we previously described the technique of successful laparoscopic removal of a TVT sling for erosion into the bladder, recurrent urinary tract infections, and pain in 5 patients. ${ }^{6}$ The sling was successfully removed in those patients with no intraoperative complications, hospital stay of $<24$ hours, and rapid recovery. This small series demonstrated laparoscopy to be a viable approach to removing the retropubic portion of the TVT sling when necessary.

Other articles have discussed a laparoscopic approach to sling removal. Rigaud et al. ${ }^{4}$ reported a transperitoneal laparoscopic approach for removal of the TVT mesh from the retropubic space in patients suffering from chronic pelvic and perineal pain. In their study, the arms of the mesh sling on either side were removed from the posterior surface of the rectus abdominis muscle to the lateral border of the urethra, and the hemicircumferential portion of mesh on the posterior surface of the urethra was left in place. This prospective study showed that $68 \%$ of patients were pain free within 10 months after sling removal, and only $22 \%$ risked the recurrence of urinary incontinence. Misrai et al. ${ }^{3}$ reported on 30 patients with retropubic mesh tape sling complications who underwent a laparoscopic extraperitoneal complete tape removal 6 to 80 months postinsertion. At 6 months postexcision, all patients reported a total decrease in pain. Roupret et al. ${ }^{5}$ used either an intra- or extraperitoneal laparoscopic approach for complete tape resection/ removal in 38 women with TVT-related complications. Fifteen out of the 48 cases were for isolated chronic pelvic pain. All women reported a decrease of symptom-related complications with mean follow-up of 38 months. Recurrent incontinence occurred in $65 \%$ of the women. However, none of these articles have addressed treatment of persistent symptoms after partial removal of the sling.

In the current case, we describe our laparoscopic technique for removal of the remaining retropubic portion of the mesh in a patient for whom previous removal of the vaginal portion of the sling had failed to resolve persistent pain. This case shows that laparoscopy is a safe surgical method to access the space of Retzius and locate the remaining mesh, even in patients with previous history of surgery. Therefore, in patients who have had previous transvaginal sling excision without improvement in symptoms, laparoscopic removal of the remaining retropubic limbs should be considered as a treatment option.

\section{References}

1. Costantini E, Lazzeri M, Porena M. Managing complications after midurethral sling for stress urinary incontinence. $E A U-E B U$ Update Series. 2007;5:232-240.

2. Latthe PM, Foon R, Toozs-Hobson P. Transobturator and retropubic tape procedures in stress urinary incontinence: a systematic review and meta-analysis of effectiveness and complications. BJOG. 2007;114:522-531.

3. Misrai V, Roupret M, Xylinas E, et al. Surgical resection for suburethral sling complications after treatment for stress urinary incontinence. J Urol. 2009;181:2198-2203.

4. Rigaud J, Pothin P, Labat J, et al. Functional results after tape removal for chronic pelvic pain following tension-free vaginal tape or transobturator tape. J Urol. 2010;184:610-615.

5. Roupret M, Misrai V, Vaessen C, Cour F, Haertig A, ChartierKastler E. Laparoscopic surgical complete sling resection for tension-free vaginal tape-related complications refractory to firstline conservative management: a single-centre experience. Eur Urol. 2010;58:270-274.

6. Pikaart DP, Miklos JR, Moore RD. Laparoscopic removal of pubovaginal polypropylene tension-free tape slings. JSLS. 2006; 10:220-225.

7. Duckett JR, Jain S. Groin pain after a tension free vaginal tape or similar suburethral sling: management strategies. BJU Int. 2005;95:95-97.

8. Fisher HW, Lotze PM. Nerve injury locations during retropubic sling procedures. Int Urogynecol J Pelvic Floor Dysfunct. 2011;22:439-441.

9. Rardin CR, Rosenblatt PL, Kohli N, Miklos JR, Heit M, Lucente VR. Release of tension-free vaginal tape for the treatment of refractory postoperative voiding dysfunction. Obstet Gynecol. 2002;100:898-902. 